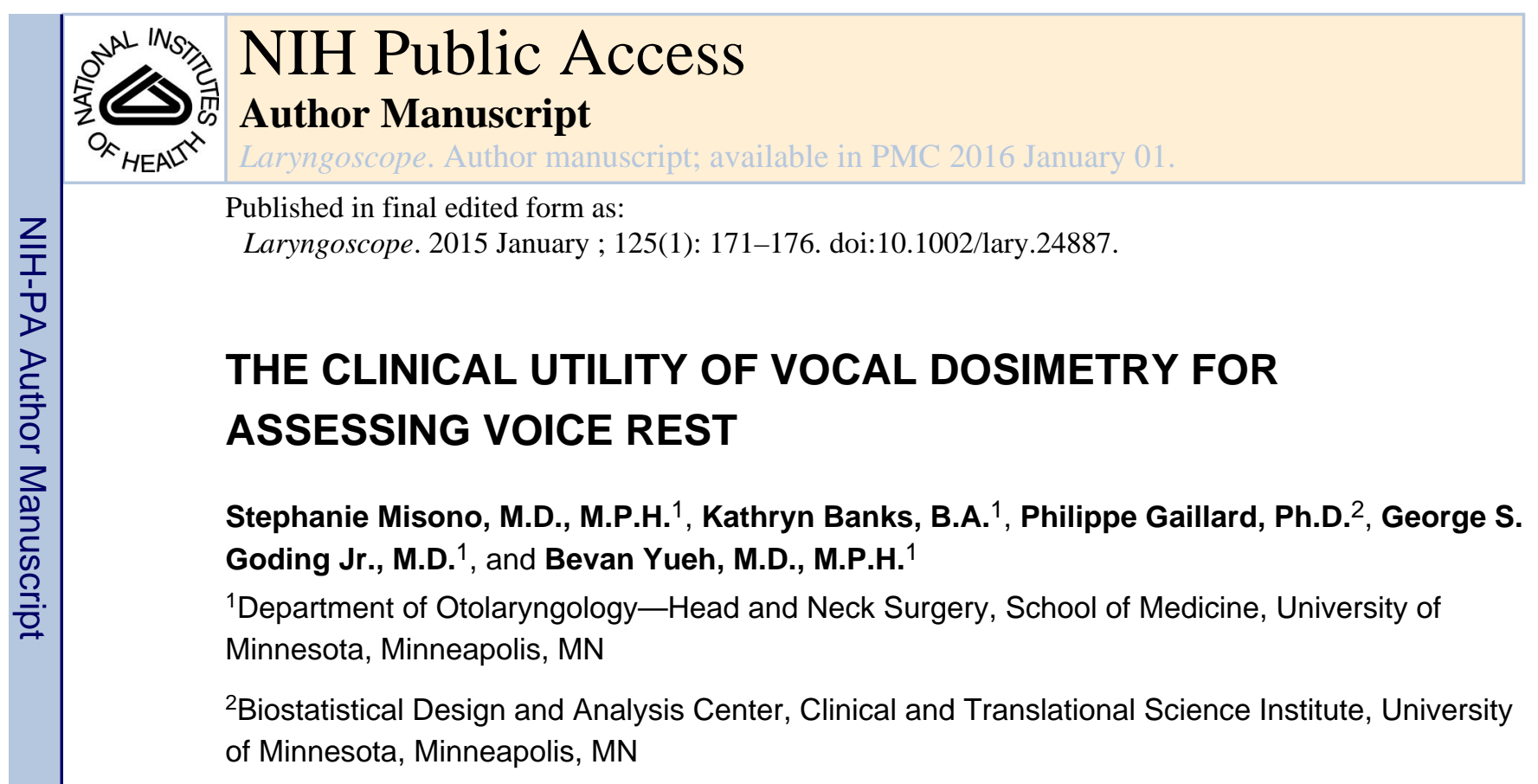

\title{
Abstract
}

Objective-Voice rest is frequently recommended following surgical disruption of vocal fold epithelium, but patients report variable adherence to voice rest recommendations. The objective of this study was to assess the clinical utility of an ambulatory vocal dosimeter for measuring adherence to voice rest recommendations.

Study Design-Outcomes research

Methods-Part 1: To determine the utility of the dosimeter in non-clinical use, the relationship between self-reported voice use and dosimeter measurements was examined in normal subjects $(n=11)$ who prospectively logged voice use while wearing the dosimeter. Part 2: To determine clinical utility of the dosimeter, patients undergoing vocal fold surgery for which post-operative voice rest was recommended $(\mathrm{n}=11)$ wore a dosimeter for two days prior to and two days after surgery. Phonation percent and sound level were compared at baseline and during voice rest.

Results-The dosimeter performed as hypothesized with both normal subjects and patients. A moderate correlation $(\mathrm{r}=0.62)$ was noted between self-reported voice use and dosimeter measurements in normal subjects. In patients on voice rest, a statistically and clinically significant decrease was observed in measured voice use, both in phonation time $(\mathrm{p}=0.002)$ and intensity of phonation $(\mathrm{p}=0.004)$.

Conclusions-Ambulatory vocal dosimetry may have clinical utility for assessing adherence to voice rest recommendations. This information will be useful for the design of future studies on voice rest.

\section{Level of evidence-2c}

Corresponding author: Stephanie Misono, Otolaryngology Department, 8th Floor, Phillips-Wangensteen Building, 420 Delaware Street S.E., MMC 396, Minneapolis, MN 55455, smisono@umn.edu, (612) 626-5526 phone, (612) 625-2101 fax.

Financial disclosures: None

Conflict of Interest: None

Presented as a poster ( $2^{\text {nd }}$ place poster prize) at Annual Meeting of the American Laryngological Association at the Combined Otolaryngology Spring Meetings, May 15, 2014, Las Vegas NV. 


\section{Keywords}

voice; voice rest; laryngeal surgery; vocal dosimeter; vocal accumulator; adherence; compliance

\section{Introduction}

The recommendation for voice rest after vocal fold surgery is debated among otolaryngologists and speech-language pathologists, but a majority of laryngologists routinely prescribe a regimen of voice rest after phonomicrosurgery despite a lack of evidence-based guidelines as to degree of voice rest or duration ${ }^{1}$. Proponents suggest that post-operative voice use may lead to continued injury to the already surgically injured vocal fold, while others propose that phonation may promote better healing by minimizing the risk of fibrosis ${ }^{2}$. The evidence supporting the use of voice rest is limited ${ }^{3}$, in part because there have been few tools to measure the degree to which patients adhere to voice rest.

A meaningful study of voice rest would necessitate compliance with recommended parameters during that period of time. Rousseau et al. have described relatively low selfreported "complete compliance" of $35 \%$ among patients who were prescribed voice rest ${ }^{4}$. An additional $44 \%$ reported that they "almost never" used their voice. Although selfreported degree of voice use was evaluated, the amount of accrued phonation, whether through talking, coughing, or throat-clearing was not known. Thus, the varying degrees of self-reported compliance among these patients may represent a spectrum of voice use patterns, but the degree of variability is unclear. It is unknown how subjective measures such as patient perception and patient report of voice use are related to objective measures such as vocal dose accumulation. It is particularly difficult for investigators to assess adherence to the recommendation for voice rest since vocal fold surgery typically occurs in the outpatient setting.

The development of technology allowing measurement of voice use represents an exciting approach to the study of voice. ${ }^{5,6}$ An accelerometer placed along the anterior neck is used to measure vibrations from the vocal folds through the tissues of the neck ${ }^{7,8}$ and converted into sound pressure levels of speech (decibels, or $\mathrm{dB}$ ). Phonation measured in this way has been shown to be relatively insensitive to surrounding sounds. ${ }^{9}$ These findings have led to the development of portable vocal dosimetry, which allows measurement and characterization of phonation using a small sensor on the anterior neck (Figure 1). One of the challenges of studying voice rest is that even with no volitional voice use, patients may be phonating through other behaviors such as throat-clearing or coughing. Dosimetry allows quantification of all types of sound production. In addition, dosimetry records only the amount of voicing produced (in $\mathrm{dB}$ ) but not the actual content of the speech, facilitating quantification without compromising privacy.

Sophisticated ambulatory vocal dosimeters have been used previously to assess vocal production and fatigue among classical music singers ${ }^{10}$ and to examine voice use in teachers, ${ }^{11}$ but their use has not been widespread due to the need for frequent downloading of data and significant cost. More recently, a relatively inexpensive portable device called the Vocalog (GriffinLabs, Temecula, CA) was developed for the purpose of encouraging 
voice use among patients with Parkinsonism. A similar device made in Sweden was recently used in a pilot study of patients with Parkinson disease to demonstrate that biofeedback given by the device encouraged patients to raise their voice sound level. ${ }^{12}$ The same group of investigators utilized the device among teachers in Sweden to examine voice use at school and at leisure ${ }^{13}$. Their work confirmed that subjects are typically tolerant of wearing a vocal dosimeter and demonstrated inter-individual variability.

Vocal dosimeters have not previously been applied to the examination of voice rest, and no studies were available in the literature describing use of the Vocalog. Therefore, the objective of this study was to determine the clinical utility ${ }^{14}$ of this vocal dosimeter in measuring vocal behavior during prescribed voice rest.

We hypothesized that 1) a useful tool for measuring voice use and voice rest would demonstrate convergent validity ${ }^{15}$, i.e., an association between self-reported voice use and measured phonation in normal subjects. We expected a moderate strength of association based on other studies of relationships between subjective and objective ambulatory measures ${ }^{16-18}$. We also hypothesized that 2) such a tool would demonstrate significantly decreased voice use (phonation percent) and loudness (sound level) in patients on voice rest after treatment.

\section{Methods}

The Vocalog vocal dosimeter was used for this study. The device can store up to 21 days of data, avoiding the need for patients to download their own measurements. Once calibrated (which was performed per manufacturer instructions prior to use by each participant), it records estimated $\mathrm{dB}$ of phonation each second throughout the monitoring period. It detects both voicing and non-speech phonatory behaviors such as throat-clearing and coughing. ${ }^{19}$ Although it can be set to alarm if phonation is above or below a threshold, the alarm function was not used in this study. We considered measurements greater than $60 \mathrm{~dB}$ to be consistent with phonation, using the standard estimate for soft conversational speech. We defined phonation percent as the proportion of time represented by measurements that reflected phonation. The numerator was the number of measurements greater than $60 \mathrm{~dB}$, and the denominator was the total number of measurements. We defined sound level as the $\mathrm{dB}$ level recorded by the device during phonation; only readings greater than $60 \mathrm{~dB}$ were included.

\section{Part 1: Vocal dosimetry and reported voice use}

Healthy adult subjects with no reported voice problems wore the vocal dosimeter and simultaneously documented their voice use over a 2 hour period. The subjects were asked to record the percentage of time spent talking, rounded to the nearest 5\%, every 15 minutes. The relationship between self-reported voice use and dosimeter measurement of phonation percent was examined using a Pearson correlation. The Bland-Altman method was used to assess for systematic bias. ${ }^{20,21}$ 
Part 2: Perioperative voice use

Patients undergoing a laryngeal procedure for which at least two days of voice rest was recommended were invited to participate. The patients were instructed to wear the device for two days prior to their procedure and the first two days following the procedure. They underwent laryngeal procedures in the OR or in clinic, and were prescribed varying durations of voice rest depending on the recommendation of the surgeon. Patients were instructed to avoid any voice use, including speaking, shouting, singing, whispering, throatclearing, and/or coughing. They were also given multiple opportunities to ask any clarifying questions pre-operatively, both when meeting with their surgeon and when meeting with their speech pathologist. Phonation percent and voice sound levels were recorded preoperatively and post-operatively and compared using paired Student's t-test.

To attempt to minimize observer bias, minor deception was used and patients were not told that the device would be measuring voice use; it was described instead as a device that measured neck muscle activity. Patients were asked to wear the device as much as possible during their participation, but were asked not to wear the device while sleeping or bathing for safety reasons. Patients were also asked not to wear the device while in a motor vehicle both for safety reasons and because pilot testing demonstrated that vibrations could be recorded by the device. Patients kept a log of when they wore the device and were also asked to push a marker button the device when putting on or taking off the device. To further minimize observer bias, we did not ask patients to log adherence to voice rest recommendations while they wore the device. When patients returned for follow-up, we asked about overall adherence to voice rest.

The study was approved by the IRB of the University of Minnesota (\# 1111M06933).

\section{Results}

\section{Part 1: Vocal dosimetry and reported voice use}

Eleven normal subjects participated in this portion of the study. Data from two subjects were not included in the analysis; one was excluded because the subject reported near-total silence throughout the recording period, making it impossible to use the data recorded by the device to determine whether reported changes in voice use were associated with changes in dosimeter measurements. Data from the other subject were excluded due to device malfunction, in which the device, when routinely tested after data collection had already been completed for this subject, was noted to be reporting high decibels even when worn in silence by research staff. This device was therefore removed from use for the remainder of the study, and the data from this subject were excluded to avoid making inaccurate conclusions secondary to this malfunction. The strength of association among the remaining 9 individual participants varied from 0.06 to 0.93 . In the aggregate, the association was $\mathrm{r}=0.62$ (scatter plot shown in Figure 2). The Bland-Altman method was used to confirm the absence of systematic bias. 


\section{Part 2: Perioperative voice use}

Eleven of fourteen eligible patients consented to participate in the study. Reasons given for not wanting to participate included distance to the clinic for a patient who could not stay to enroll that day, desire to avoid wearing a device around the neck, and difficulty managing an additional device in a patient who wears a prosthetic arm that straps around the neck.

Enrolled patients included 5 women and 6 men who ranged in age from 34 to 69 years and presented with a variety of laryngeal pathologies (Table 1). All patients who enrolled were able to use the dosimeter as instructed and no complications or problems were reported. No patients reported complete adherence to voice rest, but nearly all (10/11) patients reported observing voice rest most of the time, and one reported that it was difficult to observe voice rest.

The number of measurements for each patient as well as pre- and post- procedure phonation percents are reported in Table 2. The phonation percent was $29 \%$ pre-procedure (baseline) and $12 \%$ post-procedure (voice rest), representing a reduction of voice use by about $70 \%$. This difference was statistically significant $(\mathrm{p}=0.002)$. In 10 of 11 patients, a significant reduction in average sound level was observed during the post-procedure voice rest period (Table 3). The average decrement in the overall cohort was 66.9 to $64.5 \mathrm{~dB}(\mathrm{p}=0.004)$.

\section{Discussion}

These findings support our hypotheses that a device with clinical utility would have convergent validity with voice use in normal subjects and reflect decreased voice use in patients adherent to voice rest recommendations.

\section{Part 1: Vocal dosimetry and reported voice use}

In normal subjects, prospectively collected data on voice use was associated with vocal dosimeter measurements. Because the self-reported data were collected every 15 minutes, we anticipated that there would be minimal recall bias. We therefore expected associations with the dosimeter measurements, if the dosimeter was functioning well, to be reasonably strong. As expected, the strength of association was moderate ${ }^{22}$. The wide range in correlation coefficients across individual subjects suggests that some individuals may be more consistent and/or accurate in their self-assessment of voice use than others. This is in keeping with other aspects of the medical literature in which patient self-report was related to, but not equivalent to, objective measures ${ }^{23}$. It is also consistent with findings in other areas in which relative changes were more accurately assessed than absolute levels ${ }^{24}$.

A challenge in the examination of the relationship between self-report and dosimeter measurements is that there is no established gold standard for recording voice use that would be practical to implement clinically. The variable quality of self-reporting that we observed makes a case for the use of this or another ambulatory vocal dosimeter in combination with patient self-report. 


\section{Part 2: Perioperative voice use}

As might be expected in a heterogeneous group of patients, baseline (pre-procedure) phonation proportions were widely varied. We observed that during prescribed voice rest, patients significantly decreased voice. The decrease in phonation percent was large, indicating that the reduction was not just statistically significant but also clinically meaningful. The majority of patients (6 of 11) decreased their phonation proportion to less than $10 \%$, indicating near total silence; one patient was essentially silent, spending less than $1 \%$ of the time phonating. Conversely, the remaining 5 patients had phonation measurements recorded more frequently, with only 2 patients demonstrating voice use more than $25 \%$ of the time. Thus in this sample, the majority of patients demonstrated minimal voice use, but there was some variability in degree of reduction in vocal behavior despite the same voice rest instructions for all subjects.

There are no established guidelines for how much phonation is "compliant" to voice rest recommendations. The results reported here may be useful as a starting point for comparison. Our findings suggest that absolute silence is likely unrealistic in clinical practice, but near-total quiet was achieved by a majority of patients. Recent work from other investigators has demonstrated that the Vocalog device may overestimate phonation time, ${ }^{19}$ so although there was variability, most patients were perhaps even more adherent to the recommendation for voice rest than the reported percentages might suggest.

We observed that average sound level was significantly decreased. The mean $\mathrm{dB}$ difference of 2.36 roughly corresponds to a halving of the loudness of voice use. This implies that even when patients used their voices during the prescribed voice rest period, they were careful to modulate their volume. We set the threshold for measurements considered to be phonation at $60 \mathrm{~dB}$; this level was selected as it is standard for soft conversational speech. It is conceivable that setting the threshold lower could have captured additional phonation, but doing so would have increased the risk of misattributing as voice use any background readings due to jostling of the neck piece or other minor events. To minimize the impact of the "phonation" cutoff, each patient served as his or her own control, allowing us to comparatively assess the differences in phonation at baseline versus while on voice rest.

This study suggests that an inexpensive ambulatory dosimeter of this nature could play a valuable role in studies of voice rest, but there were limitations that need to be acknowledged. The sample sizes for this study were small, so larger studies are needed to confirm generalizability. Second, the Vocalog device does not record fundamental frequency, and therefore a precise evaluation of the number of vibratory cycles and associated phonatory impact is precluded ${ }^{7,25}$. It is also not known to what extent wearing the device affected patient behavior. It is probable that patients were aware of the presence of the device ${ }^{12}$, which could potentially affect patterns of vocal behavior, but it would not be possible to collect the measurements otherwise. There are also some potential issues specific to the device design that may influence the quality of data collection. This includes potential dislodgment of the sensor should the patient have prominent sternocleidomastoid muscles, given the relative stiffness of the collar (future versions of the device are reportedly in development). However, this limitation would be consistent across a given patient, allowing meaningful comparisons to be made between pre- and post-procedure measures. Another 
potential limitation is that because we only asked patients to wear the device for two days before and after their procedures, we do not know how patients would have responded to wearing the device for longer, nor do we know how adherent they would have been to voice rest recommendations beyond the first two days. However, patients tolerated the device well, suggesting that longer time frames may be realistic in future studies. Voice outcomes were not examined in relationship to degree of adherence to voice rest recommendations, because many of the patients' diagnoses were potentially or likely recurrent.

Despite these limitations, our findings supported the hypotheses that vocal dosimetry readings would be associated with self-reported voice use and that decreased voice use would be measured when patients were prescribed voice rest. These observations suggest clinical utility for vocal dosimetry in the evaluation of voice rest. A detailed comparison of the device used in this study to others that are currently commercially available, such as the Kay-Pentax APM and the VoxLog, is beyond the scope of this study, but each has specific characteristics that should be taken into account when selecting a device for future studies. The area of ambulatory vocal dosimetry is exciting and rapidly changing, and new generations of devices may allow better accuracy, more comprehensive data collection, lower costs, and increased ease of use for application in future studies of voice rest.

\section{Conclusions}

We observed the hypothesized moderate association between self-reported voice use and measured phonation percent. In patients prescribed voice rest, we observed a dramatic decrease in voice use, both in phonation percent and sound level. This study was a necessary step in establishing the feasibility of ambulatory vocal dosimetry in the study of voice rest, facilitating future clinical research on the impact of voice rest on vocal outcomes.

\section{Acknowledgments}

The gracious assistance of our partners in the Lions Voice Clinic, Deirdre D. Michael, PhD CCC-SLP, and Lisa Butcher, MM, MA, CCC-SLP, is gratefully acknowledged. Lisa Henn, PhD, also provided statistical input. The work was supported by funding from the Lions Club of Minnesota and NIH UL1TR000114. The authors have no financial or other relationships with GriffinLabs, and the devices used in this study were purchased by us for research purposes.

\section{References}

1. Behrman A, Sulica L. Voice rest after microlaryngoscopy: current opinion and practice. Laryngoscope. 2003; 113:2182-2186. [PubMed: 14660924]

2. Ishikawa K, Thibeault S. Voice Rest Versus Exercise: A Review of the Literature. J Voice. 2009

3. Koufman J, Blalock D. Is voice rest never indicated? Journal of voice : official journal of the Voice Foundation. 1989; 3:87-91.

4. Rousseau B, Cohen S, Zeller A, Scearce L, Tritter A, Garrett C. Compliance and quality of life in patients on voice rest. Otolaryngology Head and Neck Surgery. 2010 In Press.

5. Cheyne HA, Hanson HM, Genereux RP, Stevens KN, Hillman RE. Development and testing of a portable vocal accumulator. J Speech Lang Hear Res. 2003; 46:1457-1467. [PubMed: 14700368]

6. Ryu S, Komiyama S, Kannae S, Watanabe H. A newly devised speech accumulator. ORL J Otorhinolaryngol Relat Spec. 1983; 45:108-114. [PubMed: 6341919]

7. Hillman RE, Heaton JT, Masaki A, Zeitels SM, Cheyne HA. Ambulatory monitoring of disordered voices. Ann Otol Rhinol Laryngol. 2006; 115:795-801. [PubMed: 17165660] 
8. Svec JG, Titze IR, Popolo PS. Estimation of sound pressure levels of voiced speech from skin vibration of the neck. J Acoust Soc Am. 2005; 117:1386-1394. [PubMed: 15807026]

9. Svec, JG.; Titze, I.; Popolo, PS. Vocal dosimetry: theoretical and practical issues. In: Schade, G.; Muller, F.; Wittenberg, T.; Hess, M., editors. Proceeding Papers for the Converence Advances in Quantitative Laryngology, Voice and Speech Research. Hamburg, Germany: IRB Verlag; 2003.

10. Carroll T, Nix J, Hunter E, Emerich K, Titze I, Abaza M. Objective measurement of vocal fatigue in classical singers: a vocal dosimetry pilot study. Otolaryngol Head Neck Surg. 2006; 135:595602. [PubMed: 17011424]

11. Hunter EJ, Titze IR. Variations in intensity, fundamental frequency, and voicing for teachers in occupational versus nonoccupational settings. J Speech Lang Hear Res. 2010; 53:862-875. [PubMed: 20689046]

12. Schalling E, Gustafsson J, Ternstrom S, Bulukin Wilen F, Sodersten M. Effects of tactile biofeedback by a portable voice accumulator on voice sound level in speakers with Parkinson's disease. J Voice. 2013; 27:729-737. [PubMed: 24050819]

13. Szabo Portela A, Hammarberg B, Sodersten M. Speaking fundamental frequency and phonation time during work and leisure time in vocally healthy preschool teachers measured with a voice accumulator. Folia Phoniatr Logop. 2013; 65:84-90. [PubMed: 24107540]

14. Smart A. A multi-dimensional model of clinical utility. Int J Qual Health C. 2006; 18:377-382.

15. Campbell DT, Fiske DW. Convergent and Discriminant Validation by the Multitrait-Multimethod Matrix. Psychol Bull. 1959; 56:81-105. [PubMed: 13634291]

16. Aboras Y, El-Banna M, El-Magraby R, Ibrahim A. The relationship between subjective self-rating and objective voice assessment measures. Logopedics Phoniatrics Vocology. 2010; 35:34-38.

17. Decalmer SC, Webster D, Kelsall AA, McGuinness K, Woodcock AA, Smith JA. Chronic cough: how do cough reflex sensitivity and subjective assessments correlate with objective cough counts during ambulatory monitoring? Thorax. 2007; 62:329-334. [PubMed: 17101736]

18. Lauderdale DS, Knutson KL, Yan LL, Liu K, Rathouz PJ. Self-reported and measured sleep duration: how similar are they? Epidemiology (Cambridge, Mass). 2008; 19:838-845.

19. Searl J, Dietsch A. Testing of the VocaLog Vocal Monitor. J Voice. 2014

20. Bland JM, Altman DG. Measuring agreement in method comparison studies. Statistical methods in medical research. 1999; 8:135-160. [PubMed: 10501650]

21. Bland JM, Altman DG. Statistical methods for assessing agreement between two methods of clinical measurement. Lancet. 1986; 1:307-310. [PubMed: 2868172]

22. Taylor R. Interpretation of the Correlation-Coefficient - a Basic Review. J Diagn Med Sonog. 1990; 6:35-39.

23. Cassidy CM, Rabinovitch M, Schmitz N, Joober R, Malla A. A comparison study of multiple measures of adherence to antipsychotic medication in first-episode psychosis. Journal of clinical psychopharmacology. 2010; 30:64-67. [PubMed: 20075650]

24. Dieltjens M, Braem MJ, Vroegop AV, et al. Objectively measured vs self-reported compliance during oral appliance therapy for sleep-disordered breathing. Chest. 2013; 144:1495-1502. [PubMed: 23928873]

25. Nacci A, Fattori B, Mancini V, et al. The use and role of the Ambulatory Phonation Monitor (APM) in voice assessment. Acta Otorhinolaryngol Ital. 2013; 33:49-55. [PubMed: 23620641] 


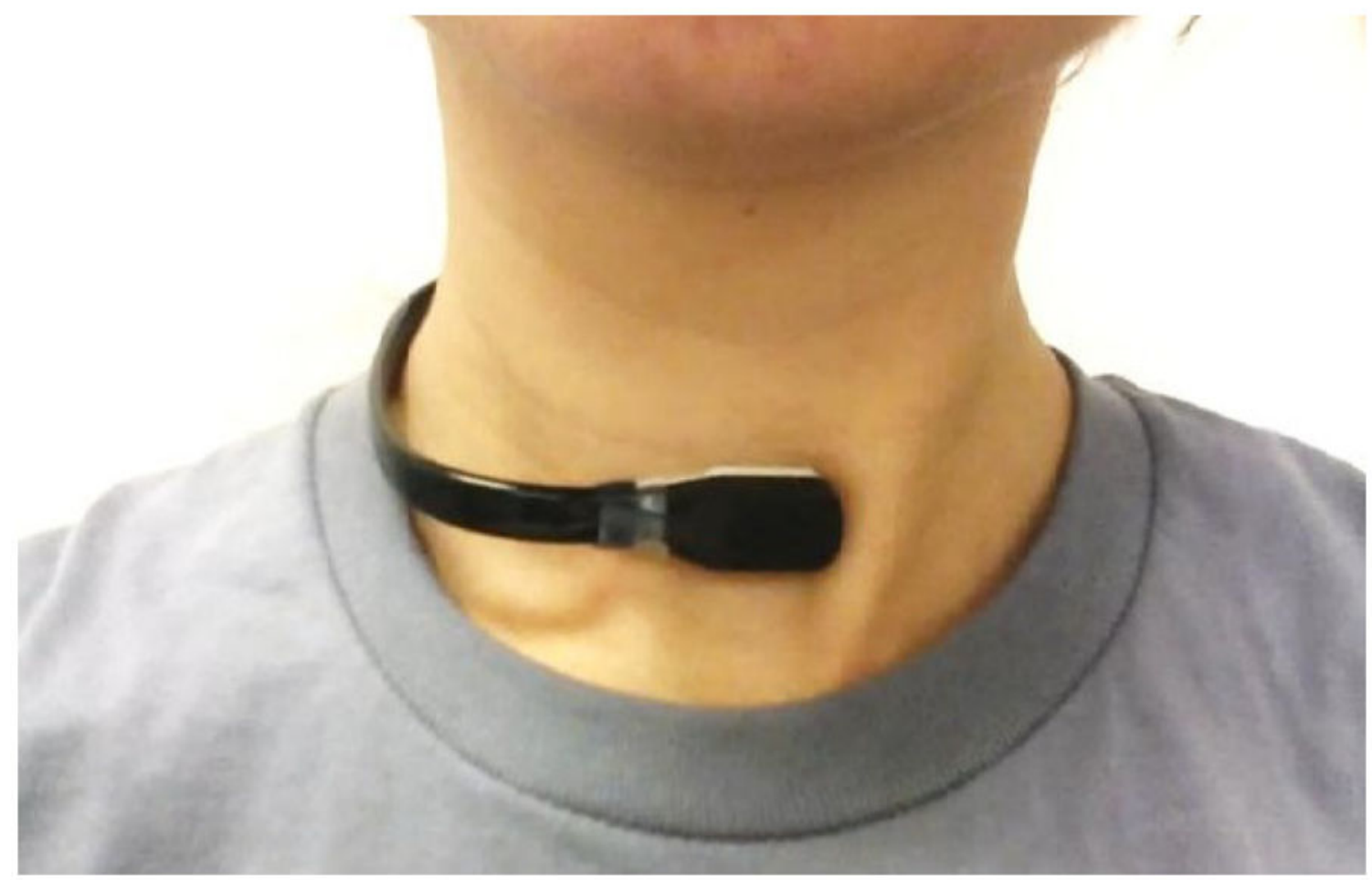

Figure 1.

Low profile neck piece for vocal dosimeter 


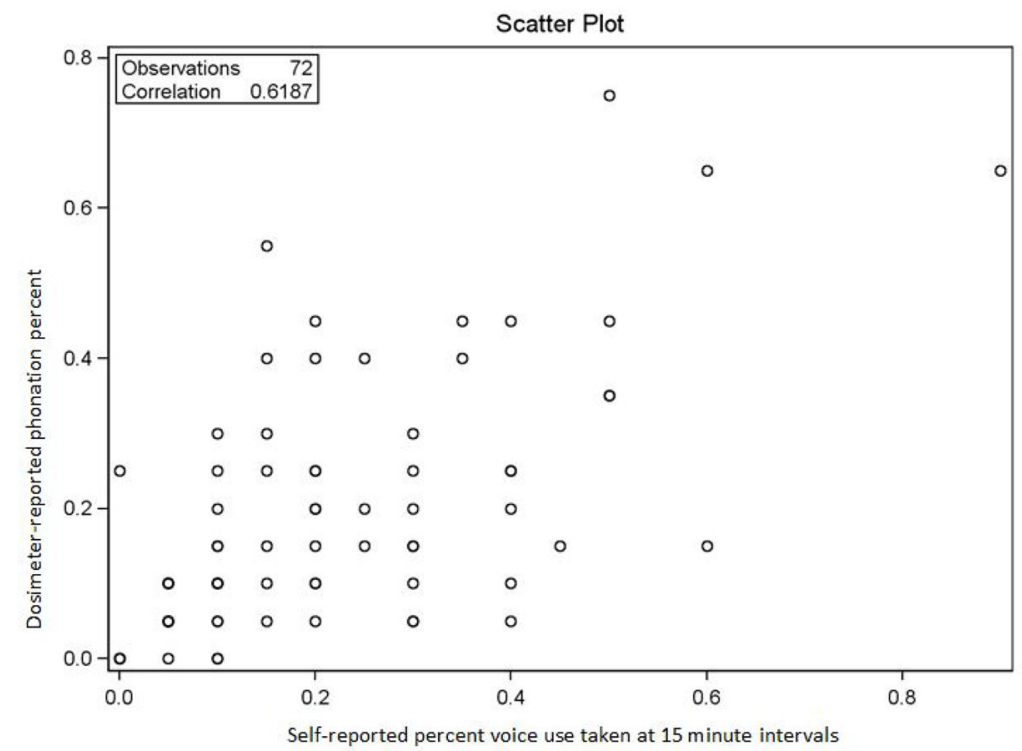

Figure 2.

Association between self-reported voice use and dosimeter measurements 


\section{Table 1}

\section{Patient characteristics}

\begin{tabular}{|c|c|c|c|l|}
\hline Subject & Age & Gender & Race & Diagnosis \\
\hline 1 & 34 & F & White & Vocal fold polyp \\
\hline 2 & 50 & F & White & Leukoplakia \\
\hline 3 & 51 & F & White & Leukoplakia \\
\hline 4 & 64 & F & White & Reinke's edema \& laryngeal lesions \\
\hline 5 & 61 & M & African-American & Recurrent squamous cell carcinoma, true vocal fold \\
\hline 6 & 58 & M & White & Carcinoma in situ, vocal fold \\
\hline 7 & 34 & M & White & Persistent vocal process granuloma, vocal fold paresis \\
\hline 8 & 69 & M & White & Recurrent respiratory papillomatosis \\
\hline 9 & 40 & M & White & Recurrent respiratory papillomatosis \\
\hline 10 & 42 & M & White & Recurrent respiratory papillomatosis \\
\hline 11 & 43 & F & Not given & Squamous cell carcinoma, true vocal fold \\
\hline
\end{tabular}


Table 2

Comparison of pre- vs post-procedure vocal dosimeter measurements: proportion of measurements with phonation

\begin{tabular}{|c|c|c|c|c|}
\hline ID & Number of measurements & Pre-procedure phonation percent & Post-procedure phonation percent & Ratio (post/pre) \\
\hline 1 & 244140 & $36.7 \%$ & $11.4 \%$ & 0.3117 \\
2 & 231299 & 39.6 & 26.5 & 0.6689 \\
3 & 159180 & 77.4 & 54.5 & 0.7047 \\
4 & 119400 & 37.5 & 14.8 & 0.3944 \\
5 & 140160 & 9.9 & 1.2 & 0.1188 \\
6 & 158760 & 22.7 & 15.2 & 0.6671 \\
7 & 219900 & 9.4 & 0.3 & 0.0403 \\
8 & 181020 & 22.4 & 4.6 & 0.2045 \\
9 & 209341 & 13.1 & 1.6 & 0.1195 \\
10 & 149640 & 20.3 & 2.8 & 0.1387 \\
11 & 238020 & 30.5 & 2.6 & 0.0845 \\
\hline & Mean (SD) & $29.0(19.4)$ & $12.3(16.2)$ & $0.31[95 \%$ CI $0.14,0.49]$ \\
& & & & \\
\hline
\end{tabular}


Table 3

Comparison of pre- vs post-procedure vocal dosimeter measurements: measured dB during phonation

\begin{tabular}{|c|c|c|c|c|}
\hline ID & N & Pre-procedure dB mean (SD) & Post-procedure dB mean (SD) & Mean difference (dB) \\
\hline 1 & 244140 & $68.21(4.55)$ & $63.41(4.14)$ & -4.80 \\
2 & 231299 & $67.89(7.13)$ & $64.97(4.95)$ & -2.92 \\
3 & 159180 & $72.04(6.53)$ & $68.74(5.90)$ & -3.30 \\
4 & 119400 & $66.09(4.07)$ & $63.17(3.51)$ & -2.92 \\
5 & 140160 & $63.77(3.26)$ & $64.23(3.44)$ & 0.46 \\
6 & 158760 & $63.45(3.61)$ & $62.95(3.22)$ & -0.50 \\
7 & 219900 & $67.66(5.39)$ & $66.68(6.29)$ & -0.98 \\
8 & 181020 & $65.92(4.38)$ & $64.82(5.06)$ & -1.10 \\
9 & 209341 & $65.31(3.65)$ & $64.73(4.95)$ & -0.58 \\
10 & 149640 & $66.43(3.64)$ & $63.83(3.65)$ & -2.60 \\
11 & 238020 & $69.06(4.08)$ & $62.34(3.10)$ & -6.72 \\
\hline & Mean (SD) & $66.9(2.5)$ & &
\end{tabular}

